\title{
Research on the Measurement of Displacement based on Grating Reticle Image
}

\author{
LI Hai-hua \\ Department of Mechanical and Electrical Engineering \\ HuaZhong University of Science and Technology \\ WenHua College \\ Wuhan, China \\ lihaihua80@163.com
}

\author{
FAN Juan \\ Department of Mechanical and Electrical Engineering \\ HuaZhong University of Science and Technology \\ WenHua College \\ Wuhan, China \\ jounkofj@yahoo.com.cn
}

\begin{abstract}
A new method is present to get movement displacement directly when applying grating reticle to AC servo driving control system to carry on high precise orientation for the micro-objects. Adjusting the optical path until grating reticle image can be gathered clearly by vidicon. After analyzing and processing the grating reticle image collected by vidicon, the mapping coefficient can be accurately calculated between grid width and pixels. Comparing the fore-and-aft two grating reticle images after the worktable moved. The phase difference of two images can be figured out by phase difference ratio arithmetic based on Lissajous figures. Accordingly the actual displacement of the worktable can be calculated by the mapping coefficient. The experiments show that this method can implement sub-pixel measurement easily and rapidly in micro electronics manufacture.
\end{abstract}

Keywords-displacement measurement; grating; image processing; Lissajous figure; AC servo motor

\section{INTRODUCTION}

With the development of micro fabrication technology, especially the integration of optoelectronics products continues to improvement, its components are decreased in size. The positioning accuracy of automatic control system was put forward higher requirements in processing and mounting of these components. It requires the control system develop into digital control system, high precision, high speed, high performance[1,2]. In the AC servo drive control system, the function of $\mathrm{AC}$ servo motor is convert the control voltage signal and phase signal into mechanical movement, which is to process the received electrical signal into a speed or angular displacement of the motor. So the key of the automatic control systems is the accuracy of controlling the position use the motor[3]. The grating reticle and image processing are applied to $\mathrm{AC}$ servo driving control system for high precision displacement measurement for the micro-objects in this paper.

\section{GRATING RETICLE IMAGE ACQUISITION}

The grating is a device incised with a number of evenly spaced grid line, it has the advantages of high precision, fast response speed, non-contact measurement and is suitable for use as a standard reticle[4,5]. This paper selects the grating with grid spacing of $0.02 \mathrm{~mm}$ as standard reticle to gather image First of all, the grating is fixed in a two-dimensional precision motion control platform, the grating moving is considered as the remove of the work table. Then determine the optical system parameters, such as the lighting system, image distance, focal length, etc. Ensuring the grating and the optical path vertically, and the camera can gather the bright and dark grating stripes through optical path. Then adjusting the optical path until getting clear grating stripes image, as shown in Figure 1. At the same time, fixing the optical path and maintain it during the experiment[6,7].

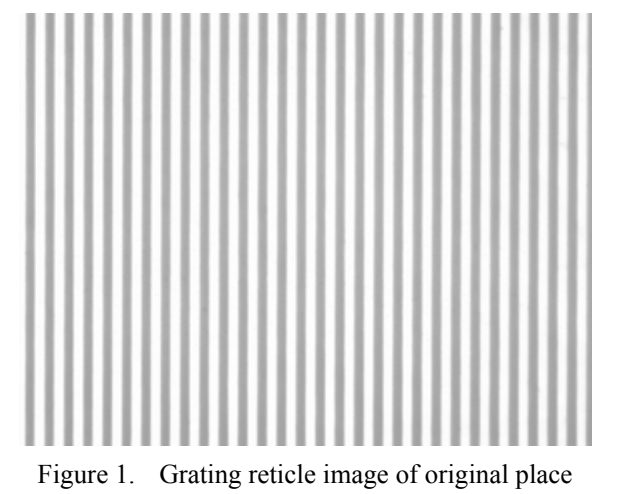

\section{IMPLEMENT OF DISPLACEMENT MEASUREMENT}

After processing and calculating for the collected grating image, the small displacement of the measured object can be acquired directly, the specific method is as follows.

\section{A. Gray value processing of the image}

In order to obtain the periodic change of the grating reticle data, this paper adopts the method of calculating the gray value to handle the reticle image. Assuming the pixel of the whole image is $M \times N$, establishing coordinate system take the upper left corner of the image as origin. Then the coordinates of any point in the image can be denoted as $(i, j)$, which gray value is $G_{i j}$. Let $I_{j}=\sum_{i=0}^{M} G_{i j}$, which represents the sum of grayscale values of all pixels in the $j$ column. Then we can draw a gray value curve which varying periodically as approximate sinusoidal function as shown in figure 2 . 


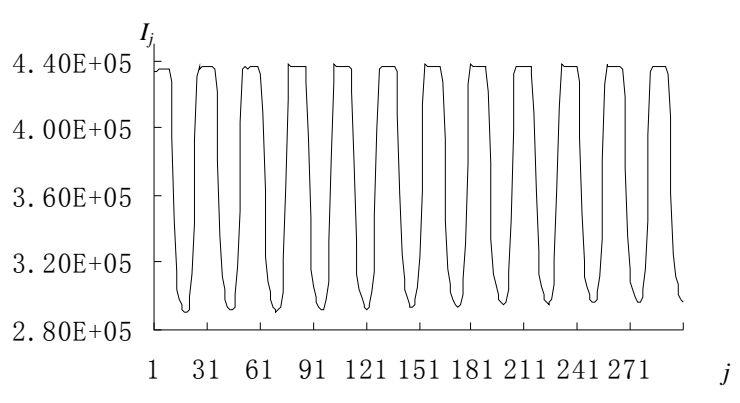

Figure 2. Gray value curve of grating reticle image

\section{B. Reticle pixel cycle calculation}

As the working table is moving, the stripe gathered by the vidicon moves along. If you want to calculate the actual displacement of the working table, you need to determine how many pixels the stripe moved when the grating moves a grid. This paper uses Lissajous figure for the corresponding relationship between grid spacing and the cycle of image pixels[8]. Let

$$
\left\{\begin{array}{l}
X_{j}=I_{j} \\
Y_{j}=I_{j+d}
\end{array} \quad(1 \leq j \leq N-d)\right.
$$

$I_{j}$ and $I_{j+d}$ represents the sum of grayscale values of all pixels in the $j$ column fore-and-aft the working table moves $d(0 \leqslant d \leqslant \mathrm{~N})$ pixels. Supposing a grid spacing of grating corresponds to $k$ pixels in the image, then $X_{j}$ and $Y_{j}$ can be seen as two signal source with period of $k$ pixels. Drawing the Lissajous figure of grating image grayscale value in $X_{j}, Y_{j}$ abscissa ordinate, as shown in figure 3.

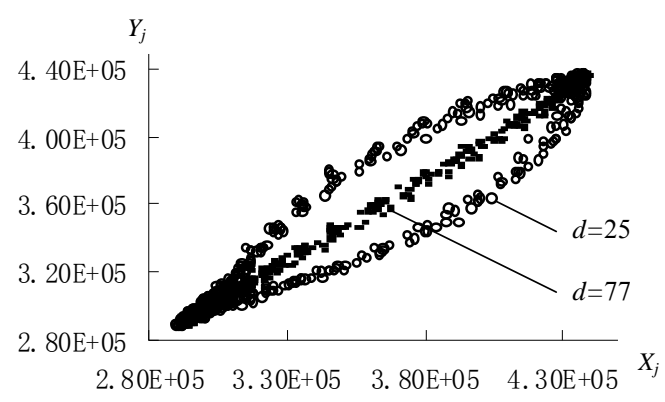

Figure 3. Lissajous figure for seeking $\mathrm{k}$

As can be seen, Lissajous figure changes constantly along with the different values of $d$. When the selected value of $d$ closer to integer multiple of the grating pixels period $k$, Lissajous figure is closer to the beeline $Y_{j}=X_{j}$. When the selected value of $d$ keep away from integer multiple of $k$, Lissajous figure becomes the shape of ellipse. Selecting the value of $d$ and the number of pixel period $w$ when Lissajous figure closest to the straight line. Looking the calculated $k$ values as the pixel cycle of grating reticle stripe, then

$$
k=d / w
$$

Taking multiple values of $d$ and $w$ to test, drawing the Lissajous figure when $d=25, w=1$ and $d=77, w=3$, as shown in figure 3 . The figure shows that when $d=77$ Lissajous figure is closest to a straight line. Then figure out $k \approx 25.667$, which is the pixel cycle of the grating stripes.

The mapping between the table displacement and the pixel width of the image is calculated according to the pixel cycle of the grating stripes. Assume the workbench actual move of displacement is $X$ when the image of grating reticles moving one pixel width, then

$$
x=20 / k=20 / 25.667 \approx 0.779 \mu \mathrm{m}
$$

\section{Calculation for the displacement}

In order to accurately calculate the pixel motion of grating image, Lissajous Figure phase ratio algorithm is adopted when the grating stripes collected from moving[9]. Specific methods are as follows:

1) Drawing the fore-and-aft gray value curve of move in the same coordinate system according the gray values of two grating images gathered by vidicon, as shown in figure 4 . The solid line represents the gray value curve of the grating gathered before move, and the dashed represents the gray value curve after the grating move. Compare the two curves can be seen, the stripes of bright and dark got a positive move.

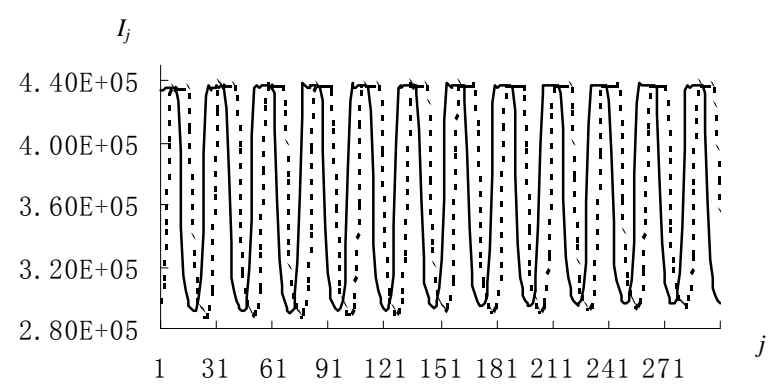

Figure 4. Contrast of fore-and-aft gray value curve

2) Using the similar method to the front, the Lissajous figure for the gray value of the grating image can be drawn as $X_{j}$ is the abscissa and $Y_{j}$ is the vertical axis, shown in Figure 5.

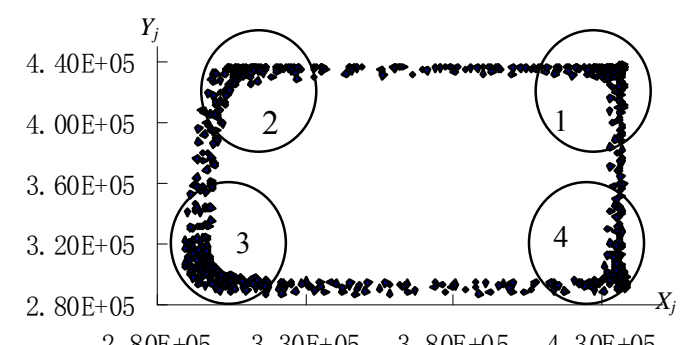

Figure 5. Lissajous figure 
In Figure 5, the region 1 and 3 represent the gray value of the corresponding point is very close between the $j-I_{j}$ curves for the two images, namely the overlapping region of the bright and dark stripes. But the region 2 and 4 represent the gray value of the corresponding point has large difference between the $j-I_{j}$ curves for the two images, namely the moving parts of the stripes. Moreover, the region 2 represents the points which are dark stripes before move and become to bright stripes after move. The region 4 represents the points which are bright stripes before move and become to dark stripes after move.

Particularly, when the phase difference of $X_{j}$ and $Y_{j}$ is $2 n \pi$, the points on the Lissajous figure are all located in region 1 and 3, and showing a straight line with a slope of 1 . When the phase difference of $X_{j}$ and $Y_{j}$ is $(2 n+1) \pi$, the points on the Lissajous figure are all located in region 2 and 4 , and showing a straight line with a slope of -1 .

Analyzing the points in a cycle, we found the grating moving to the positive direction when the points in figure 5 appear counterclockwise, whereas the grating moving to the negative direction.

3) Selecting $m$ consecutive points in Figure 4, $m$ is an integer multiple of the pixel cycle. The average gray value of the $m$ consecutive points can be calculated.

$$
\bar{I}=\frac{1}{2 m} \sum_{j=1}^{m}\left(X_{j}+Y_{j}\right)
$$

The four regions in Figure 5 can be marked off by the boundary $\bar{I}$. Taking count of the number of pixels $m_{2}$ and $m_{4}$ in region 2 and 4 respectively, then working out $\bar{m}=\left(m_{2}+m_{4}\right) / 2$, which is the total number of pixels that stripes have moved.

4) According to the proportion of the $\bar{m}$ pixels in the total number of pixels, the number of pixels that grating move really can be calculated.

$$
n=k \times \bar{m} / m
$$

Then the displacement of the work table can be figured out.

$$
S=n x
$$

\section{EXPERIMENTS AND ERROR ANALYSIS}

The AC servo motor is used for driving and controlling the move of two dimension exact motive platform in experiments. The control system is mainly composed of the host computer, controller, driver, execute motor, optical encoder and power supply, etc. The software is programmed by Visual $\mathrm{C}++6.0$. Input speed value, displacement and direction information through keyboard, the computer can pass the position command signal to the motor drive after interpolation calculation. Let the pulse signal to control the steps that the motor will go, and the direction signals to control motor rotation forward and reverse. The servo motor is driven by drives and work according to the required action. The computer receives the feedback signal generated by the optical encoder at the same time. Consequently, the detection and controlling for speed and displacement of the screw driven by the servo motor comes true, and the position control for two-axis is implemented.

The following analysis and calculation a set of image that gathered after motor turned twenty positive pulses. When the drive controller sends out twenty pulses to the motor, the work table should move $4 \mu \mathrm{m}$ ideally toward the positive direction. Figure 4 shows the fore-and-aft gray value curve when the work table moved. Selecting 693 consecutive points from the figure which include all the entire cycles of stripes, then

$$
\begin{aligned}
& \bar{I}=\sum_{j=1}^{693}\left(X_{j}+Y_{j}\right) /(2 \times 693) \approx 3.6964 \times 10^{5} \\
& m_{2}=142, m_{4}=145, \bar{m}=\left(m_{2}+m_{4}\right) / 2=143.5 \\
& n=25.667 \times \bar{m} / 693 \approx 5.315 \text { pixel } \\
& S=n x \approx 4.140 \mu \mathrm{m}, \text { it is the displacement. }
\end{aligned}
$$

Subsequently, the dual-frequency laser interferometer is used to measure the mobile displacement and the measurement result is regarded as the actual displacement. Comparing the value of $\mathrm{S}$ with the actual displacement and figuring out the error $\delta$. Repeat the above test procedure, the displacement measurement test data can be gained, as shown in Table 1.

TABLE I. EXPERIMENT DATA OF DISPLACEMENT MEASURING

\begin{tabular}{|c|c|c|c|c|c|c|}
\hline $\begin{array}{c}\text { Test } \\
\text { number }\end{array}$ & $\begin{array}{c}\bar{I}(\times \\
\mathbf{1 0})\end{array}$ & $\bar{m}$ & $n$ (pixel) & $S\left(\mu_{\mathrm{m}}\right)$ & $\begin{array}{c}\text { Actual } \\
\text { shift } \\
\left(\mu_{\mathrm{m}}\right)\end{array}$ & $\delta\left(\mu_{\mathrm{m}}\right)$ \\
\hline 1 & 3.6964 & 143.5 & 5.315 & 4.140 & 3.62 & 0.520 \\
\hline 2 & 3.6662 & 145.5 & 5.389 & 4.198 & 3.76 & 0.438 \\
\hline 3 & 3.6400 & 138.5 & 5.130 & 3.996 & 4.20 & 0.204 \\
\hline 4 & 3.6738 & 113.5 & 4.204 & 3.275 & 3.76 & 0.485 \\
\hline 5 & 3.7063 & 115 & 4.260 & 3.319 & 3.45 & 0.131 \\
\hline 6 & 3.7429 & 131.5 & 4.870 & 3.794 & 4.03 & 0.236 \\
\hline 7 & 3.8242 & 167 & 6.185 & 4.818 & 4.72 & 0.098 \\
\hline
\end{tabular}

The experimental data in Table 1 are processed to calculate the average measurement error is $\bar{\delta}=\sum_{i=1}^{7} \delta_{i} / 7 \approx 0.3 \mu \mathrm{m}$. This method can obtain sub-pixel measurement accuracy[10].

\section{CONCLUSION}

A new method for measuring motion displacement is put forward in this paper by applying grating reticles to the highprecision positioning servo drive control system for the microobject. It gets high accuracy when we measure for the motion displacement of the two-dimensional precision motion control platform. The experiments show that this method can implement sub-pixel measurement easily and rapidly in micro electronics manufacture. 


\section{REFERENCES}

[1] L. Nancy, H. Breck, "Advances in lasers on a chip," Photonics Spectra, vol. 39, pp. 76-80, September 2005.

[2] HOU Jie, QIAN Jiaru, and ZHANG Weijing, "2-D displacement measurement system based on image processing," Journal of Tsinghua University (Science and Technology), vol. 50, pp. 826-829, June 2010.

[3] HOU Shujuan, WU Siliang, "Measurement of miss distance for targets with known modes of motion," Transaction of Beijing Institute of Technology, vol. 25, pp. 625-627, July 2005.

[4] R. Giuliana, "Grey level image components for multi-scale representation," Lecture Notes in Computer Science, 3287, pp. 574-579, 2004.

[5] LI Haihua, YANG Liangen, and WANG Xuanze, "A method of getting displacement directly using standard reticle and CCD," China Mechanical Engineering, vol. 18, pp. 2881-2883, December 2007.
[6] LI Penghui, ZHAO Wenguang, and ZHU Hongping, "Monitoring multidynamic displacements by digital image processing technology," Journal of Huazhong University of Science and Technology. Nature Science, vol. 39, pp. 80-84, February 2011.

[7] XIANG Li, ZHANG Lin, and HE Wei, "Two-dimensional tiny displacement measurement based on SOPC and image processing," Applied Science and Technology, vol. 35, pp. 9-12, February 2008.

[8] M. Chantler, "The effect of illuminant rotation on texture filters: Lissajous's ellipses," Lecture Notes in Computer Science, 2352, pp. 289295, 2002.

[9] DING Kang, "Time-shifting correcting method of phase difference on discrete spectrum," Applied Mathematics and Mechanics, vol. 23, pp. 819-827, July 2002.

[10] H. Nobach, D. Nils, and T. Cam, "High-precision sub-pixel interpolation in particle image velocimetry image processing," Experiments in Fluids, vol. 39, pp. 299-304, February 2005. 